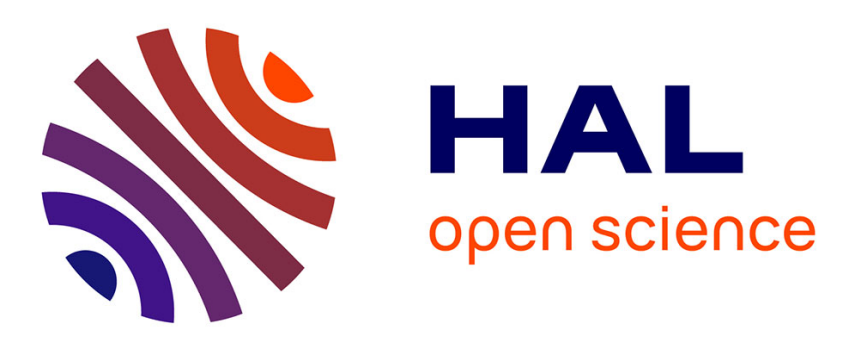

\title{
The Hardness and Approximation of the Densest k-Subgraph Problem in Parameterized Metric Graphs
}

Shih-Chia Chang, Li-Hsuan Chen, Ling-Ju Hung, Shih-Shun Kao, Ralf Klasing

\section{To cite this version:}

Shih-Chia Chang, Li-Hsuan Chen, Ling-Ju Hung, Shih-Shun Kao, Ralf Klasing. The Hardness and Approximation of the Densest k-Subgraph Problem in Parameterized Metric Graphs. 2020 International Computer Symposium (ICS), Dec 2020, Tainan, Taiwan. pp.126-130, 10.1109/ICS51289.2020.00034 . hal-03441672

\author{
HAL Id: hal-03441672 \\ https://hal.science/hal-03441672
}

Submitted on 22 Nov 2021

HAL is a multi-disciplinary open access archive for the deposit and dissemination of scientific research documents, whether they are published or not. The documents may come from teaching and research institutions in France or abroad, or from public or private research centers.
L'archive ouverte pluridisciplinaire HAL, est destinée au dépôt et à la diffusion de documents scientifiques de niveau recherche, publiés ou non, émanant des établissements d'enseignement et de recherche français ou étrangers, des laboratoires publics ou privés. 


\title{
The Hardness and Approximation of the Densest $k$-Subgraph Problem in Parameterized Metric Graphs
}

\author{
Shih-Chia Chang*, Li-Hsuan Chen ${ }^{\dagger}$, Ling-Ju Hung ${ }^{\ddagger}$, Shih-Shun Kao ${ }^{\S}$ and Ralf Klasing $₫$ \\ ${ }^{*}$ Department of Business Administration, College of Management \\ National Taipei University of Business, Taipei, Taiwan \\ $\dagger$ ProBrand Technology, Inc., Taoyuan City 330, Taiwan \\ $\S \uparrow$ CNRS, LaBRI, Université de Bordeaux, Talence, France \\ $\S$ Department of Computer Science and Information Engineering \\ National Cheng Kung University, Tainan, Taiwan
}

\begin{abstract}
A complete weighted graph $G=(V, E, w)$ is called $\Delta_{\beta}$-metric, for some $\beta \geq 1 / 2$, if $G$ satisfies the $\beta$-triangle inequality, i.e., $w(u, v) \leq \bar{\beta} \cdot(w(u, x)+w(x, v))$ for all vertices $u, v, x \in V$. Given a $\Delta_{\beta}$-metric graph $G=(V, E, w)$, the $\boldsymbol{\Delta}_{\beta}$-WeIGHTED DENSEST $\boldsymbol{k}$-SUBGRAPH $\left(\boldsymbol{\Delta}_{\beta}\right.$-WD $k$ S $)$ problem is to find an induced subgraph $G[C]$ with exactly $k$ vertices such that the total edge weight of $G[C]$ is maximized. For $\beta=1$, this problem, $\Delta-\mathrm{WD} k \mathrm{~S}$, is known NP-hard and admits a $\frac{1}{2}$-approximation algorithms. In this paper, we show that for any $\beta>1 / 2, \Delta_{\beta}$-WDkS is NP-hard. We also show how to modify any $\alpha$-approximation algorithm for $\Delta$-WDkS to obtain a $\delta_{\alpha, \beta}$-approximation algorithm for $\Delta_{\beta}-\mathrm{WD} k \mathrm{~S}$ with $\delta_{\alpha, \beta}>\alpha$ for every $\beta<1$. Moreover, we prove that $\Delta_{\beta}-\mathrm{WD} k \mathrm{~S}$ can be approximated to within a factor $\frac{1}{2 \beta}$ for any $\beta>\frac{1}{2}$.
\end{abstract}

\section{INTRODUCTION}

Various real-world systems can be modeled as graph-based representation. Many applications in social networks, communication networks, mobile ad hoc networks, World Wide Web (WWW) communities, bioinformatics are related to find a dense subgraph from a large graph [26]. In particular, on studying social networks, detecting cohesive subgroups is a very important task. It helps sociologists to understand the structures of networks. A cohesive subgroup can be defined as a complete graph (clique) [37]. However, it seems too restricted to consider a clique as a cohesive subgroup in real networks. The concept dense subgraph is a density-based clique relaxation model for defining cohesive subgraphs in social networks.

Given an undirected unweighted graph $G$, a densest $k$ subgraph of $G$ is an induced subgraph $G[C]$ of $G$ with exactly $k$ vertices such that the number of edges is maximized. If $G$

This research was supported by the LaBRI under the "Projets émergents" program. This study has been carried out in the frame of the "Investments for the future" Programme IdEx Bordeaux - SysNum (ANR-10-IDEX-03-02).

*Email: chang@ntub.edu.tw

†Email: g943339@oz.nthu.edu.tw

†Email: ljhung@ntub.edu.tw (Corresponding author)

Email: shih-shun.kao@labri.fr

Email: ralf.klasing@1abri.fr is a weighted graph, a densest $k$-subgraph of $G$ is an induced subgraph $G[C]$ of $G$ having exactly $k$ vertices satisfying that the total edge weight is maximized. The concept of densest $k$-subgraph is often used to define cohesive subgroups in a social network. In the following, we list the formal definition of the DENSEST $k$-SUBGRAPH problem.

DENSEST $k$-SUBGRAPH PROBLEM $(\mathrm{D} k \mathrm{~S})$

Input: An undirected graph $G=(V, E)$, an integer $k>0$. Output: A vertex subset $C \subseteq V,|C|=k$ such that the number of edges in $G[C]$ is maximized.

WEIGHTED DENSEST $k$-SUBGRAPH PROBLEM (WD $k \mathrm{~S}$ )

Input: An undirected weighted $G=(V, E, w)$, an integer $k>0$.

Output: A vertex subset $C \subseteq V,|C|=k$ such that the total edge weight of $G[C]$ is maximized.

Known results. A densest $k$-subgraph is also called a $k$ cluster [25]. The problem of finding a densest $k$-subgraph in an undirected graph was introduced by Corneil and Perl [25]. It is a generalization of the maximum clique problem. The $\mathrm{D} k \mathrm{~S}$ problem is NP-hard on general graphs [25] and remains NP-hard on chordal graphs [25], bipartite graphs [25], planar graphs [32], even on graphs of maximum degree three [27]. Some exact exponential time algorithms were given for solving the $\mathrm{D} k \mathrm{~S}$ problem in general graphs [17], [18].

It has been shown that the $\mathrm{D} k \mathrm{~S}$ problems does not admit a Polynomial Time Approximation Scheme (PTAS) for general graphs under a complexity assumption [33]. There are PTASes given for graphs of minimum degree $\Omega(n)$ and dense graphs (of $\Omega\left(n^{2}\right)$ edges) when $k$ is $\Omega(n)$ [3], stars of cliques [35] and interval graphs [39]. Many approximation algorithms were developed for the $\mathrm{D} k \mathrm{~S}$ problem on general graphs and special graphs. Feige et al. gave an approximation algorithm with approximation ratio $O\left(n^{\delta}\right)$, for some $\delta<\frac{1}{3}$ for the $\mathrm{D} k \mathrm{~S}$ problem on general graphs [28]. Bhaskara et al. improved the ratio to be $O\left(n^{1 / 4+\epsilon}\right)$ for any $\epsilon>0$ [7]. Asahiro et al. presented a simple greedy algorithm for this problem on general graphs 
and showed that the approximation ratio is $O(n / k)$ [4]. Chen et al. gave constant factor approximation algorithms for a large family of intersection graphs [19]. In [36], Liazi et al. gave a 3approximation algorithm for chordal graphs. Backer and Keil gave a $\frac{3}{2}$-approximation algorithm for proper interval graphs and bipartite permutation graphs [5]. For $\mathrm{WD} k \mathrm{~S}$, it was shown NP-hard for metric graphs [40]. There are two approximation algorithms with approximation factors 4 [40] and 2 [29] for the $\mathrm{WD} k \mathrm{~S}$ problem in metric graphs.

In this paper, we focus on solving the $\mathrm{WD} k \mathrm{~S}$ problem in $\Delta_{\beta}$-metric graphs. A complete weighted graph $G=(V, E, w)$ is called $\Delta_{\beta}$-metric, for some $\beta \geq 1 / 2$, if $w(u, v) \geq 0$ for $u, v \in V$, and $G$ satisfies the $\beta$-triangle inequality, i.e., $w(u, v) \leq \beta \cdot(w(u, x)+w(x, v))$ for all vertices $u, v, x \in V$. For $\beta=1$, it defines the so-called metric graphs. The formal problem definition is listed in the following.

\section{$\Delta_{\beta}$-Weighted DenSEST $k$-SUBGRAPH PROBLEM} $\left(\Delta_{\beta}-\mathrm{WD} k \mathrm{~S}\right)$

Input: $\quad$ A $\Delta_{\beta}$-metric graph $G=(V, E, w)$, an integer $k>$ 0 .

Output: A vertex subset $C \subseteq V,|C|=k$ such that $w(C)=$ $\sum_{u, v \in C} w(u, v)$ is maximized.

For $\beta=1$, i.e., the input graph is a metric graph, we use $\Delta$-WD $k \mathrm{~S}$ to denote $\Delta_{1}$-WD $k \mathrm{~S}$.

The design of approximation algorithms for the $\Delta-\mathrm{WD} k \mathrm{~S}$ problem is related to the concept of stability of approximation for hard optimization problems [11], [15], [30], [31], [34]. It is similar to that of the stability of numerical algorithms. Suppose there is a small change in the specification (some parameters, characteristics) of the set of problem instances. It is of interesting to see that what the approximation ratio would be changed accordingly. We say an algorithm is stable if the change of the approximation ratio is small for every small change in the set of problem instances. There have been many research results on the concept of stability of approximation for solving fundamental hard optimization problems. E.g. in [1], [2], [6], [9]-[12], [38] it was shown that one can partition the set of all input instances of the Traveling Salesman Problem into infinitely many subclasses according to the degree of violation of the triangle inequality, and for each subclass one can guarantee upper and lower bounds on the approximation ratio. Similar studies demonstrated that the $\beta$-triangle inequality can serve as a measure of hardness of the input instances for other problems as well, in particular for the problem of constructing 2-connected spanning subgraphs of a given complete edge-weighted graph [13], and for the problem of finding, for a given positive integer $k \geq 2$, and an edge-weighted graph $G$, a minimum $k$-edge- or $k$-vertexconnected spanning subgraph [14], [16]. Moreover, $\beta$-triangle inequality is also applied to measure the hardness of several hub allocation problems [20]-[23].

In Section II, we prove that for any $\beta>\frac{1}{2}$, the $\Delta_{\beta}$ $\mathrm{WD} k \mathrm{~S}$ problem is NP-hard. In Section III, we show how to modify any $\alpha$-approximation algorithm for $\Delta$-WD $k \mathrm{~S}$ to obtain a $\delta_{\alpha, \beta}$-approximation algorithm for $\Delta_{\beta}$-WD $k \mathrm{~S}$ with $\delta_{\alpha, \beta}>\alpha$ for every $\beta<1$. In Section IV, we show that a $\frac{1}{2}$ approximation algorithm given in [29] for solving the WD $k \mathrm{~S}$ problem in metric graphs can be applied to solve the $\Delta_{\beta}$ $\mathrm{WD} k \mathrm{~S}$ problem for any $\beta>\frac{1}{2}$ and the approximation ratio is $\frac{1}{2 \beta}$. The concluding remarks are given in Section V.

We close this section with some notation and definitions. For a vertex subset $C$ of a weighted graph $G=(V, E, w)$, we use $w(C)$ to denote the total edge weight of $G[C]$, i.e., $w(C)=$ $\sum_{u, v \in C} w(u, v)$. We use $n$ to denote the number of vertices in a graph $G$. The approximation ratio used in this paper is $\frac{A P X}{O P T}$ where $A P X$ is the size of the approximation solution and $O P T$ is the size of the optimal solution. Notice that the $\Delta_{\beta}$-WD $k \mathrm{~S}$ problem is a maximization problem, $\frac{A P X}{O P T} \leq 1$.

\section{NP-HARDNESS}

In this section, we prove that for $\beta>\frac{1}{2}$, the $\Delta_{\beta}$-WD $k \mathrm{~S}$ is NP-hard. This shows that even in subclasses of metric graphs $\beta<1$ (e.g., $\beta=\frac{1}{2}+\epsilon$ for any $0<\epsilon<\frac{1}{2}$ ), the $\Delta_{\beta}$-WD $k \mathrm{~S}$ is still NP-hard.

Theorem 1. For any $\beta>\frac{1}{2}$, the $\Delta_{\beta}$-WDkS problem is NPhard.

Proof. We prove that the $\Delta_{\beta}$-WD $k \mathrm{~S}$ problem is at least as hard as the NP-hard problem, the $\mathrm{D} k \mathrm{~S}$ problem.

For an input graph $G=(V, E)$ of the $\mathrm{D} k \mathrm{~S}$ problem, construct a $\Delta_{\beta}$-metric graph $G^{\prime}=(V, E, w)$ such that $w(u, v)=2 \beta$ if $(u, v) \in E$, otherwise $w(u, v)=1$. It is easy to see that $G^{\prime}$ is a $\Delta_{\beta}$-metric graph satisfying the $\beta$ triangle inequality for $\beta \geq \frac{1}{2}$. We show that the $\Delta_{\beta}$-WD $k \mathrm{~S}$ problem is as hard as the $\mathrm{D} k \mathrm{~S}$ problem.

Let $C$ be an optimal solution of the $\Delta_{\beta}-\mathrm{WD} k \mathrm{~S}$ problem in $G^{\prime}$ and $w(C)=2 \beta \cdot p+\left(\begin{array}{c}k \\ 2\end{array}\right)-p$, i.e., $G^{\prime}[C]$ has $p$ edges with weight $2 \beta$ and $\left(\left(\begin{array}{l}k \\ 2\end{array}\right)-p\right)$ edges with weight 1 . Since the edge cost in $G^{\prime}$ is either $2 \beta$ or 1 , we see that $G[C]$ has exactly $p$ edges. Suppose that there exists a vertex subset $D$ of size $k$ such that $G[D]$ has more than $p$ edges. It is easy to see that in $G^{\prime}, w(D)>2 \beta \cdot p+\left(\begin{array}{c}k \\ 2\end{array}\right)-p$, a contradiction. Thus, if $C$ is an optimal solution of the $\Delta_{\beta}$-WD $k \mathrm{~S}$ problem in $G^{\prime}$, then $C$ is an optimal solution of the $\mathrm{D} k \mathrm{~S}$ problem in $G$. Notice that $w(u, v) \geq 0$ for $u, v \in V$ since $G$ satisfies the $\beta$-triangle inequality.

By the fact that the $\mathrm{D} k \mathrm{~S}$ problem is an NP-hard problem, this implies that the $\Delta_{\beta}$-WD $k \mathrm{~S}$ problem is also an NP-hard problem. This completes the proof.

Remark 1. Theorem 1 shows that the $\Delta_{\beta}$-WDkS problem is already NP-hard on the class of $\Delta_{\beta}$-metric graphs where all the edge costs are in $\{1,2 \beta\}$.

\section{Using $\Delta$-WD $k \mathrm{~S}$ APPROXIMATION Algorithms FOR $\Delta_{\beta}-\mathrm{WD} k \mathrm{~S}$}

In this section we show how to modify any $\alpha$-approximation algorithm for $\Delta$-WD $k \mathrm{~S}$ to obtain a $\delta_{\alpha, \beta}$-approximation algorithm for $\Delta_{\beta}$-WDkS with $\delta_{\alpha, \beta}>\alpha$ for every $\beta<1$. The advantage of this approach is that any improvement on the approximation of $\Delta-\mathrm{WD} k \mathrm{~S}$ automatically results in an 
improvement of the approximation ratio for $\Delta_{\beta}-\mathrm{WD} k \mathrm{~S}$. The idea of this approach is to reduce an input instance of $\Delta_{\beta}$ $\mathrm{WD} k \mathrm{~S}$ to an input instance of $\Delta-\mathrm{WD} k \mathrm{~S}$ by subtracting a suitable cost from all edges.

Lemma 1 ( [9]). Let $G$ be a $\Delta_{\beta}$-metric graph for $\frac{1}{2} \leq \beta<1$. Let $c_{\min }$ and $c_{\max }$ be the minimum edge cost and maximum edge cost in $G$ respectively. Then $c_{\max } \leq \frac{2 \beta^{2}}{1-\beta} \cdot c_{\min }$.

Theorem 2. Let $A$ be an approximation algorithm for $\Delta$ WDkS with approximation ratio $\alpha$, and let $\frac{1}{2}<\beta<1$. Then $A$ is an approximation algorithm for $\Delta_{\beta}-W D k S$ with approximation ratio $^{1} \alpha+(1-\alpha) \cdot \frac{(1-\beta)^{2}}{\beta^{2}}$.

Proof. Let $I=(G$, cost $)$ be a problem instance of $\Delta_{\beta}-\mathrm{WD} k \mathrm{~S}$, $\frac{1}{2}<\beta<1$. Let $c=(1-\beta) \cdot 2 \cdot c_{\min }$ where $c_{\min }$ is the minimum edge cost in $G$. For all $e \in E(G)$, let $\operatorname{cost}^{\prime}(e)=\operatorname{cost}(e)-c$. Then the WD $k \mathrm{~S}$ instance $I^{\prime}=\left(G\right.$, cost $\left.^{\prime}\right)$ still satisfies the triangle inequality: Let $x, y, z$ be the costs of the edges of an arbitrary triangle of $G$. Then $z \leq \beta \cdot(x+y)$ holds. Since

$$
c=(1-\beta) \cdot 2 \cdot c_{\min } \leq(1-\beta) \cdot(x+y)
$$

it follows that $z \leq \beta \cdot(x+y) \leq x+y-c$ and thus

$$
z-c \leq(x-c)+(y-c) .
$$

Furthermore we know that a $k$-subgraph is optimal for $I^{\prime}$ if and only if it is optimal for $I$. Let $H_{\mathrm{opt}}$ be an optimal $k$-subgraph for $I$. Let $H$ be the $k$-subgraph that is produced by the algorithm $A$ on the input $I^{\prime}$. Then $\operatorname{cost}^{\prime}(H) \geq \alpha$. $\operatorname{cost}^{\prime}\left(H_{\mathrm{opt}}\right)$ holds and thus

$$
\operatorname{cost}(H)-\left(\begin{array}{l}
k \\
2
\end{array}\right) \cdot c \geq \alpha \cdot\left(\operatorname{cost}\left(H_{\mathrm{opt}}\right)-\left(\begin{array}{l}
k \\
2
\end{array}\right) \cdot c\right) .
$$

This leads to

$$
\begin{aligned}
\operatorname{cost}(H) \geq & \alpha \cdot \operatorname{cost}\left(H_{\mathrm{opt}}\right)+(1-\alpha) \cdot\left(\begin{array}{l}
k \\
2
\end{array}\right) \cdot c \\
= & \alpha \cdot \operatorname{cost}\left(H_{\mathrm{opt}}\right) \\
& +(1-\alpha) \cdot\left(\begin{array}{l}
k \\
2
\end{array}\right) \cdot(1-\beta) \cdot 2 \cdot c_{\mathrm{min}} \\
\geq & \alpha \cdot \operatorname{cost}\left(H_{\mathrm{opt}}\right)+(1-\alpha) \cdot\left(\begin{array}{l}
k \\
2
\end{array}\right) \\
& \cdot(1-\beta) \cdot 2 \cdot \frac{1-\beta}{2 \beta^{2}} \cdot c_{\mathrm{max}}(\text { by Lemma } 1) \\
= & \alpha \cdot \operatorname{cost}\left(H_{\mathrm{opt}}\right) \\
& +(1-\alpha) \cdot \frac{(1-\beta)^{2}}{\beta^{2}} \cdot\left(\begin{array}{l}
k \\
2
\end{array}\right) \cdot c_{\mathrm{max}} \\
\geq & \alpha \cdot \operatorname{cost}\left(H_{\mathrm{opt}}\right) \\
& +(1-\alpha) \cdot \frac{(1-\beta)^{2}}{\beta^{2}} \cdot \operatorname{cost}\left(H_{\mathrm{opt}}\right) \\
= & \left(\alpha+(1-\alpha) \cdot \frac{(1-\beta)^{2}}{\beta^{2}}\right) \cdot \operatorname{cost}\left(H_{\mathrm{opt}}\right)
\end{aligned}
$$

which completes the proof.

\footnotetext{
${ }^{1}$ Observe that the approximation ratio tends to 1 with $\beta$ approaching $\frac{1}{2}$ and it tends to $\alpha$ with $\beta$ approaching 1 .
}

According to Theorem 2, we have the following corollary.

Corollary 1. For $\frac{1}{2} \leq \beta<1$, Algorithm 1 is $a\left(\frac{1}{2}+\frac{(1-\beta)^{2}}{2 \beta^{2}}\right)$ approximation algorithm for $\Delta_{\beta}-W D k S$.

Note that Corollary 1 provides a weaker approximation ratio than Theorem 3 in the next section.

\section{A $\frac{1}{2 \beta}$-APPROXIMATION ALGORITHM FOR ALL $\beta>\frac{1}{2}$}

In [29], a $\frac{1}{2}$-approximation algorithm was given for solving the $\mathrm{WD} k \mathrm{~S}$ problem in metric graphs. We list this algorithm in Algorithm 1. In this section, we show that Algorithm 1 can be applied to solve the $\Delta_{\beta}$-WD $k \mathrm{~S}$ problem for any $\beta>\frac{1}{2}$ and the approximation ratio is $\frac{1}{2 \beta}$. It means that the algorithm can be applied to solve the problem not only restricted to the input graph being a metric graph but also in a graph belonging to a super graph class of metric graphs.

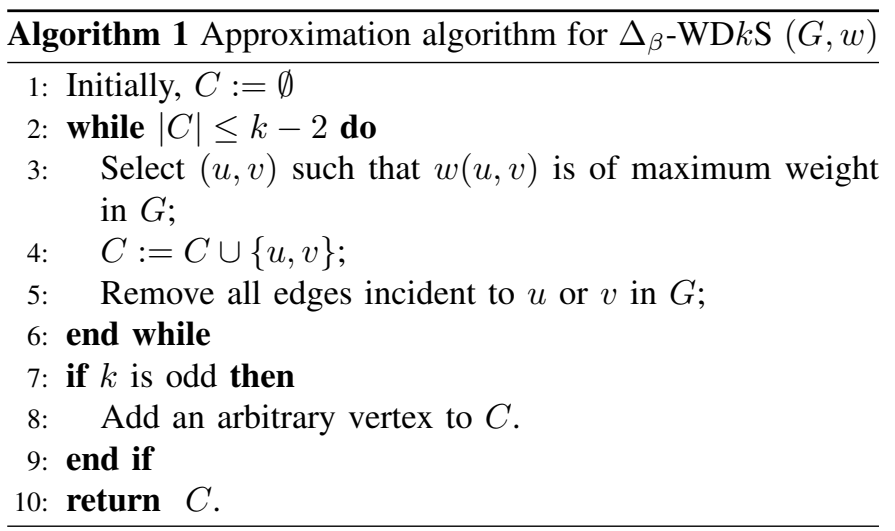

Theorem 3. For $\beta \geq \frac{1}{2}$, the $\Delta_{\beta}$-WDkS problem can be approximated to within a factor $\frac{1}{2 \beta}$ in $O\left(n^{2}+k^{2} \log k\right)$ time.

Proof. Let $C_{k}$ be the solution returned by Algorithm 1 for the $\Delta_{\beta}-\mathrm{WD} k \mathrm{~S}$. Let $C_{k}^{*}$ be an optimal solution of the $\Delta_{\beta}$ $\mathrm{WD} k \mathrm{~S}$ problem in $G$. Let $e=(u, v)$ be the edge of maximum weight in $G$ and let $G^{\prime}=G[V \backslash\{u, v\}]$. Let $C_{k-2}$ be the approximation solution on $G^{\prime}$ returned by Algorithm 1 . Assume that $C_{k-2}=C_{k} \backslash\{u, v\}$. Let $C_{k-2}^{*}$ be an optimal solution on $G^{\prime}$. The proof is by induction on $k$.

If $k=2$, we see that

$$
\begin{aligned}
w\left(C_{2}^{*}\right)= & w(x, y) \leq w(u, v) \\
& (\text { since } w(u, v) \text { is of maximum weight in } G) \\
= & w\left(C_{2}\right) \\
\leq & 2 \beta \cdot w\left(C_{2}\right) .
\end{aligned}
$$

Thus $\frac{w\left(C_{2}\right)}{w\left(C_{2}^{*}\right)} \geq \frac{1}{2 \beta}$. The theorem is true.

Suppose that $k=3$. Let $C_{3}^{*}=\{x, y, z\}$. We see that 


$$
\begin{aligned}
w\left(C_{3}^{*}\right) & =w(x, y)+w(y, z)+w(z, x) \\
& \leq 3 \cdot w(u, v)
\end{aligned}
$$

(since $(u, v)$ is of maximum weight)

$$
\leq w(u, v)+2 \cdot \beta \cdot(w(u, t)+w(t, v))
$$

(by $\beta$-triangle inequality)

$$
\begin{gathered}
\leq \quad 2 \beta(w(u, v)+w(u, t)+w(t, v))) \\
\quad\left(\text { by } \beta \geq \frac{1}{2}\right) \\
=2 \beta \cdot w\left(C_{3}\right) .
\end{gathered}
$$

Thus, $\frac{w\left(C_{3}\right)}{w\left(C_{3}^{*}\right)} \geq \frac{1}{2 \beta}$. The theorem is true for $k \leq 3$.

Suppose that the theorem is true for $k-2$. Now we prove it for $k$. Notice that $(u, v)$ is a maximum weight edge in $G$. There are three cases.

Case 1: $u, v \in C_{k}^{*}$. Let $e=(u, v)$.

Case 2: $u \in C_{k}^{*}$ and $v \notin C_{k}^{*}$. Arbitrary pick $x \in C_{k}^{*}$ and let $e=(u, x)$.

Case 3: $u, v \notin C_{k}^{*}$. Arbitrary pick $x, y \in C_{k}^{*}$ and let $e=$ $(x, y)$.

Next we prove the ratio $\frac{w\left(C_{k}\right)}{w\left(C_{k}^{*}\right)} \geq \frac{1}{2 \beta}$.

$$
\begin{aligned}
w\left(C_{k}^{*}\right) & \leq w(e)+2(k-2) \cdot w(u, v)+w\left(C_{k-2}^{*}\right) \\
& \leq w(u, v)+2(k-2) \cdot w(u, v)+2 \beta \cdot w\left(C_{k-2}\right)
\end{aligned}
$$

(by induction hypothesis)

$$
\begin{aligned}
\leq w & (u, v)+2 \sum_{t \in C_{k-2}} \beta \cdot(w(u, t)+w(v, t)) \\
& +2 \beta \cdot w\left(C_{k-2}\right)
\end{aligned}
$$

(by $\beta$-triangle inequality)

$$
\begin{aligned}
\leq & 2 \beta \cdot\left(w(u, v)+\sum_{t \in C_{k-2}}(w(u, t)+w(v, t))\right. \\
& \quad+2 \beta \cdot w\left(C_{k-2}\right) \quad\left(\text { by } \beta \geq \frac{1}{2}\right) \\
= & 2 \beta \cdot w\left(C_{k}\right) .
\end{aligned}
$$

Thus, we obtain that $\frac{w\left(C_{k}\right)}{w\left(C_{k}^{*}\right)} \geq \frac{1}{2 \beta}$. This shows that $\Delta_{\beta^{-}}$ $\mathrm{WD} k \mathrm{~S}$ problem can be approximated to within a factor $\frac{1}{2 \beta}$.

It is not hard to see that a straightforward implementation of Algorithm 1 is $O\left(k n^{2}\right)$. It was proved in [29] that by applying a linear time selection algorithm [8] and a heap data structure [24], Algorithm 1 can be executed in $O\left(n^{2}+k^{2} \log k\right)$ time. This completes the proof.

Corollary 2. The approximation ratio $\frac{1}{2 \beta}$ of Algorithm 1 is asymptotically tight.

Proof. We give an example to show that the approximation ratio $\frac{1}{2 \beta}$ of Algorithm 1 is asymptotically tight. The example can be construted by the following steps:

1) Construct a graph $G$ of $n=4 h$ vertices, consisting of a left half $G_{L}$ and a right half $G_{R}$. Let $k=2 h$.

2) The weights in $G$ are constructed as follows: (a) Identify a perfect matching of the $2 h$ vertices in $G_{L}$, and give each of the edges of the matching weight $2 \beta$. All other edges in $G_{L}$ have weight 1 .

(b) All edges in $G_{R}$ have weight $2 \beta$.

(c) All edges between $G_{L}$ and $G_{R}$ have weight 1 .

It is not hard to see that $G$ is a $\Delta_{\beta}$-metric graph. An optimal solution of the $\Delta_{\beta}$-WDkS problem in $G$ can be obtained by selecting all vertices in $G_{R}$. We have $O P T=\left(\begin{array}{l}k \\ 2\end{array}\right) \cdot 2 \beta$. If Algorithm 1 chooses all vertices of $G_{L}$ into the solution, the solution returned will be $A P X=\left(\begin{array}{l}k \\ 2\end{array}\right)+\frac{k}{2} \cdot(2 \beta-1)$. This implies

$$
\begin{aligned}
\frac{A P X}{O P T} & =\frac{\left(\begin{array}{l}
k \\
2
\end{array}\right)+\frac{k}{2} \cdot(2 \beta-1)}{\left(\begin{array}{l}
k \\
2
\end{array}\right) \cdot 2 \beta} \\
& =\frac{1}{2 \beta}+\frac{2 \beta-1}{2 \beta} \cdot \frac{\frac{k}{2}}{\left(\begin{array}{l}
k \\
2
\end{array}\right)} \\
& \leq \frac{1}{2 \beta}+\frac{1}{k-1} \\
& \approx \frac{1}{2 \beta} \quad\left(\text { since } k=\frac{n}{2}\right) .
\end{aligned}
$$

This shows that the approximation ratio $\frac{1}{2 \beta}$ of Algorithm 1 is asymptotically tight even when the edge weights have only two distinct values.

\section{CONCLUDING REMARKS}

In this paper, we prove that for $\beta>\frac{1}{2}$, the $\Delta_{\beta}$-WD $k \mathrm{~S}$ problem is NP-hard. It implies that for $\frac{1}{2}<\beta<1$ (subclasses of metric graphs), the $\Delta_{\beta}$-WD $k \mathrm{~S}$ problem is still NP-hard. We show that a $\frac{1}{2}$-approximation algorithm given for solving the WDkS problem in metric graphs can be applied to solve the $\Delta_{\beta}$-WD $k \mathrm{~S}$ problem for any $\beta>\frac{1}{2}$ and its approximation ratio is $\frac{1}{2 \beta}$. It is of interesting to see that whether $\Delta_{\beta}-\mathrm{WD} k \mathrm{~S}$ problem can be approximated to within a factor better than $\frac{1}{2 \beta}$ for any $\beta$, especially for $\beta<1$. Moreover, it is also of interesting to know whether the $\Delta_{\beta}$-WD $k \mathrm{~S}$ problem has a PTAS. If not, we must show that there exists a function $r(\beta)$ such that to approximate the $\Delta_{\beta}-\mathrm{WD} k \mathrm{~S}$ to within a factor $r(\beta)$ is NP-hard.

\section{REFERENCES}

[1] T. Andreae: On the traveling salesman problem restricted to inputs satisfying a relaxed triangle inequality. Networks, vol. 38 (2001), pp. 59 67.

[2] T. Andreae, H.-J. Bandelt: Performance guarantees for approximation algorithms depending on parameterized triangle inequalities. SIAM Journal on Discrete Mathematics, vol. 8 (1995), pp. 1-16.

[3] S. Arora, D. Karger, and M. Karpinski, Polynimial time approximation schemes for dense instances of NP-hard problems, In Proceedings of the 27th Annual ACM Symposium on Theory of Computing, pp. 284-293, 1995.

[4] Y. Asahiro, K. Iwama, H. Tamaki and T. Tokuyama, Greedily finding a dense subgraph, Journal of Algorithms, vol. 34 (2000), pp. 203-221.

[5] J. Backer and J. M. Keil, Constant factor approximation algorithms for the densest $k$-subgraph problem on proper interval graphs and bipartite permutation graphs, Information Processing Letters, vol. 110 (2010), pp. 635-638. 
[6] M.A. Bender, C. Chekuri: Performance guarantees for the TSP with a parameterized triangle inequality. Information Processing Letters, vol. 73 (2000), pp. 17-21.

[7] A. Bhaskara, M. Charika, E. Chlamtac, U. Feige, and A. Vijayaraghavan, Detecting high log-densities: an $O\left(n^{1 / 4}\right)$-approximation algorithms for the densest $k$-subgraph, In Proceedings of the 42nd ACM Symposium on Theory of Computing (STOC'10), pp. 201-210, 2010.

[8] M. Blum, R. W. Floyd, V. R. Pratt, R. L. Rivest, and R. E. Tarjan, Time bounds for selection, Journal of Computer and System Sciences, vol. 7 (1973), pp. 448-461.

[9] H.-J. Böckenhauer, J. Hromkovič, R. Klasing, S. Seibert, W. Unger, Approximation algorithms for the TSP with sharpened triangle inequality, Information Processing Letters, vol. 75 (2000), pp. 133-138.

[10] H.-J. Böckenhauer, S. Seibert: Improved lower bounds on the approximability of the traveling salesman problem. RAIRO - Theoretical Informatics and Applications, vol. 34 (2000), pp. 213-255.

[11] H.-J. Böckenhauer, J. Hromkovič, R. Klasing, S. Seibert, W. Unger: Towards the Notion of Stability of Approximation for Hard Optimization Tasks and the Traveling Salesman Problem (Extended Abstract) Proc. CIAC 2000, LNCS 1767, Springer 2000, pp. 72-86. Full version in Theoretical Computer Science, vol. 285 (2002), pp. 3-24.

[12] H.-J. Böckenhauer, J. Hromkovič, R. Klasing, S. Seibert, W. Unger: An Improved Lower Bound on the Approximability of Metric TSP and Approximation Algorithms for the TSP with Sharpened Triangle Inequality (Extended Abstract). Proc. STACS 2000, LNCS 1770 , Springer 2000, pp. 382-394.

[13] H.-J. Böckenhauer, D. Bongartz, J. Hromkovič, R. Klasing, G. Proietti, S. Seibert, W. Unger: On the hardness of constructing minimal 2-connected spanning subgraphs in complete graphs with sharpened triangle inequality. Proc. FSTTCS 2002, LNCS 2556, Springer 2002, pp. 59-70. Full version in Theoretical Computer Science, vol. 326 (2004), pp. 137-153.

[14] H.-J. Böckenhauer, D. Bongartz, J. Hromkovič, R. Klasing, G. Proietti, S. Seibert, W. Unger: On $k$-Edge-Connectivity Problems with Sharpened Triangle Inequality. In: R. Petreschi, G. Persiano, R. Silvestri (eds.), Algorithms and Complexity, Proc. 5th Italian Conference, CIAC 2003, LNCS 2653, Springer 2003, pp. 189-200.

[15] H.-J. Böckenhauer, J. Hromkovič, S. Seibert: Stability of Approximation. In: T. F. Gonzalez (ed.): Handbook of Approximation Algorithms and Metaheuristics, Chapman \& Hall/CRC, 2007, Chapter 31.

[16] H.-J. Böckenhauer, D. Bongartz, J. Hromkovič, R. Klasing, G. Proietti, S. Seibert, W. Unger: On $k$-Connectivity Problems with Sharpened Triangle Inequality. Journal of Discrete Algorithms, vol. 6 (2008), pp. 605-617.

[17] Nicolas Bourgeois, A. Giannakos, G. Lucarelli, I. Milis, V. Th. Paschos, Exact and superpolynomial approximation algorithms for the densest $k$ subgraph problem, European Journal of Operational Research, vol. 262 (2017), pp. 894-903.

[18] M.-S. Chang, L.-H. Chen, L.-J. Hung, P. Rossmanith, G.-H. Wu, Exact algorithms for problems related to the densest $k$-set problem, Information Processing Letters, vol. 114 (2014), 510-513.

[19] D. Z. Chen, R. Fleischer, and Jian Li, Densest $k$-subgraph approximation on intersection graphs, In Proceedings of the 8th International Workshop on Approximation and Online Algorithms (WAOA'10), pp. 83-93, 2010.

[20] L.-H. Chen, S.-Y. Hsieh, L.-J. Hung, and R. Klasing, The approximability of the $p$-hub center problem with parameterized triangle inequality, Proceedings of the 23rd Annual International Computing and Combinatorics Conference (COCOON 2017), LNCS 10392, pp. 112 123.

[21] L.-H. Chen, S.-Y. Hsieh, L.-J. Hung, R. Klasing, C.-W. Lee, and B. $\mathrm{Y}$. Wu, On the complexity of the star $p$-hub center problem with parameterized triangle inequality, The 10th International Conference on Algorithms and Complexity (CIAC 2017), LNCS 10236, pp. 152-163, 2017.

[22] L.-H. Chen, D.-W. Cheng, S.-Y. Hsieh, L.-J. Hung, R. Klasing, C.-W. Lee, and B. Y. Wu, Approximability and inapproximability of the star $p$-hub center problem with parameterized triangle inequality, Journal of Computer and System Sciences, vol. 92 (2018), pp. 92-112.

[23] L.-H. Chen, S.-Y. Hsieh, L.-J. Hung, and R. Klasing, Approximation algorithms for the $p$-hub center routing problem in parameterized metric graphs, Theoretical Computer Science, vol. 806 (2020), pp. 271-280.

[24] T. H. Cormen, C. E. Leiserson, R. L. Rivest, and C. Stein, Introduction to Algorithms, The MIT Press, 2009.

[25] D. Corneil, Y. Perl, Clustering and domination in perfect graphs, Discrete Appl. Math., vol. 9 (1984), pp. 27-40.

[26] A. Faragó and Z. R. Mojaveri, In search of the densest subgraph, Algorithms, vol. 12 (2019) 157: 1-18.

[27] U. Feige and M. Seltser, On the dense $k$-subgraph problem, Technical Report, The Weizmann Institute, 1997.

[28] U. Feige, G. Kortsarz, and D. Peleg, The dense $k$-subgraph problem, Algorithmica, vol. 29 (2001), pp. 410-421.

[29] R. Hassin, S. Rubinstein, and A. Tamir, Approximation algorithms for maximum dispersion, Operations Research Letters, vol. 21 (1997), pp. 133-137.

[30] J. Hromkovič: Stability of approximation algorithms and the knapsack problem. In: J. Karhumäki, H. Maurer, G. Paun, G. Rozenberg (Eds.) Jewels are Forever, Springer 1999, pp. 238-249.

[31] J. Hromkovič: Algorithmics for Hard Problems - Introduction to Combinatorial Optimization, Randomization, Approximation, and Heuristics. Second Edition, Springer 2003

[32] J. M. Keil, and T. Brecht, The complexity of clustering in planar graphs, Journal of Combinatorial Mathematics and Combinatorial Computing, vol. 9 (1991), pp. 155-159.

[33] S. Khot, Ruling out PTAS for graph min-bisection, dense $k$-subgraph, and bipartite clique, SIAM Journal on Computing, vol. 36 (2006), pp. 1025-1071.

[34] R. Klasing and T. Mömke, A modern view on stability of approximation. In: Adventures Between Lower Bounds and Higher Altitudes - Essays Dedicated to Juraj Hromkovič on the Occasion of His 60th Birthday, LNCS 11011, pp. 393-408, 2018.

[35] M. Liazi, I. Milis, and V. Zissimopoulos, The densest $k$-subgraph problem on clique graphs, Journal of Combinatorial Optimization, vol 14 (2007), pp. 465-474.

[36] M. Liazi, I. Milis, and V. Zissimopoulos, A constant approximation algorithm for the densest $k$-subgraph problem on chordal graphs, Information Processing Letters, vol. 108 (2008), pp. 29-32.

[37] R. D. Luce and A. Perry, A method of matrix analysis of group structure. Psychometrika, vol. 14 (1949), pp. 95-116.

[38] T. Mömke, An improved approximation algorithm for the traveling salesman problem with relaxed triangle inequality. Information Processing Letters, vol. 115 (2015), pp. 866-871.

[39] T. Nonner, PTAS for densest $k$-subgraph in interval graphs, Proceedings of Workshop on Algorithms and Data Structures (WADS 2011), LNCS 6844, pp. 631-641.

[40] S. S. Ravi, D. J. Rosenkrantz, G. K. Tayi, Heuristic and special case algorithms for dispersion problems, Operations Research, vol. 42 (1994), pp. 299-310.

[41] H. Singh, M. Kumar, P. Aggarwal, Approximation of heaviest $k$ subgraph problem by size reduction of input graph. In: Krishna C., Dutta M., Kumar R. (eds), Proceedings of 2nd International Conference on Communication, Computing and Networking, Lecture Notes in Networks and Systems, vol 46, pp. 599-606, Springer, Singapore, 2019. 